\title{
VALUE DISTRIBUTION OF CERTAIN DIFFERENTIAL POLYNOMIALS
}

\author{
INDRAJIT LAHIRI
}

(Received 5 October 2000 and in revised form 1 April 2001)

\begin{abstract}
We prove a result on the value distribution of differential polynomials which improves some earlier results.
\end{abstract}

2000 Mathematics Subject Classification. 30D35.

1. Introduction and definitions. Let $f$ be a transcendental meromorphic function in the open complex plane. The problem of possible Picard values of derivatives of $f$ reduces to the problem of whether certain polynomials in a meromorphic function and its derivatives necessarily have zeros. We do not explain the standard definitions and notations of value distribution theory as those are available in [6].

DEFINITION 1.1. A meromorphic function " $a$ " is said to be a small function of $f$ if $T(r, a)=S(r, f)$.

DEFINITION 1.2 (see $[1,4,10]$ ). Let $n_{0 j}, n_{1 j}, \ldots, n_{k j}$ be nonnegative integers. The expression $M_{j}[f]=(f)^{n_{0 j}}\left(f^{(1)}\right)^{n_{1 j}} \cdots\left(f^{(k)}\right)^{n_{k j}}$ is called a differential monomial generated by $f$ of degree $\gamma_{M_{j}}=\sum_{i=0}^{k} n_{i j}$ and weight $\Gamma_{M_{j}}=\sum_{i=0}^{k}(i+1) n_{i j}$.

The sum $P[f]=\sum_{i=1}^{l} b_{j} M_{j}[f]$ is called a differential polynomial generated by $f$ of degree $\gamma_{P}=\max \left\{\gamma_{M_{j}}: 1 \leq j \leq l\right\}$ and weight $\Gamma_{P}=\max \left\{\Gamma_{M_{j}}: 1 \leq j \leq l\right\}$, where $T\left(r, b_{j}\right)=S(r, f)$ for $j=1,2, \ldots, l$.

The numbers $\underline{\gamma}_{P}=\min \left\{\gamma_{M_{j}}: 1 \leq j \leq l\right\}$ and $k$ (the highest order of the derivative of $f$ in $P[f]$ ) are called, respectively, the lower degree and order of $P[f]$.

$P[f]$ is said to be homogeneous if $\gamma_{P}=\gamma_{P}$.

Also $P[F]$ is called a quasi differential polynomial generated by $f$ if, instead of assuming $T\left(r, b_{j}\right)=S(r, f)$, we just assume that $m\left(r, b_{j}\right)=S(r, f)$ for the coefficients $b_{j}(j=1,2, \ldots, l)$.

DEFINITION 1.3. Let $m$ be a positive integer. We denote by $N(r, a ; f \mid \leq m)(N(r, a$; $f \mid \geq m)$ ) the counting function of those $a$-points of $f$ whose multiplicities are not greater (less) than $m$, where each $a$-point is counted according to its multiplicity.

In a similar manner, we define $N(r, a ; f \mid<m)$ and $N(r, a ; f \mid>m)$.

Also $\bar{N}(r, a ; f \mid \leq m), \bar{N}(r, a ; f \mid \geq m), \bar{N}(r, a ; f \mid<m)$, and $\bar{N}(r, a ; f \mid>m)$ are defined similarly, where in counting the $a$-points of $f$ we ignore the multiplicities.

Finally, we agree to take $\bar{N}(r, a ; f \mid \leq \infty) \equiv \bar{N}(r, a ; f)$ and $N(r, a ; f \mid \leq \infty) \equiv N(r, a ; f)$.

DEFINITION 1.4. For two meromorphic functions $f, g$ and positive integer $m$, we denote by $N(r, a ; f \mid g=b,>m)$ the counting function of those $a$-points of $f$, counted 
with proper multiplicities, which are the $b$-points of $g$ with multiplicities greater than $m$.

DEFINITION 1.5 (see [2]). Let $m$ be a positive integer. We denote by $N_{m}(r, a ; f)$ the counting function of $a$-points of $f$, where an $a$-point of multiplicity $\mu$ is counted $\mu$ times if $\mu \leq m$ and $m$ times if $\mu>m$.

As the standard convention, we mean by $N(r, f)$ and $\bar{N}(r, f)$ the counting functions $N(r, \infty ; f)$ and $\bar{N}(r, \infty ; f)$, respectively.

Hayman [5] proved the following theorems.

THEOREM 1.6. If $f$ is a transcendental meromorphic function and $n(\geq 5)$ is a positive integer, then $\psi=f^{\prime}-a f^{n}$ assumes all finite values infinitely often.

THEOREM 1.7. If $f$ is a transcendental meromorphic function and $n(\geq 3)$ is a positive integer, then $\psi=f^{\prime} f^{n}$ assumes all finite values, except possibly zero, infinitely often.

When $f$ is transcendental, entire conclusions of Theorems 1.6 and 1.7 hold, respectively for $n \geq 3$ (cf. [5]) and $n \geq 1$ (cf. [3]).

To study the value distribution of differential polynomials Yang [7] proved the following results.

THEOREM 1.8. Let $f$ be a transcendental meromorphic function with $N(r, f)=$ $S(r, f)$, and let $\psi=f^{n}+P[f]$, where $n(\geq 2)$ is an integer and $P[f]$ is a differential polynomial generated by $f$ with $\gamma_{P} \leq n-2$. Then $\delta(a ; \psi)<1$ for $a \neq 0, \infty$.

THEOREM 1.9. Let $f$ be a transcendental meromorphic function with $N(r, f)=$ $S(r, f)$, and let $\psi=f^{n} P[f]$, where $n(\geq 2)$ is an integer and $P[f]$ is a differential polynomial generated by $f$. Then $\delta(a ; \psi)<1$ for $a \neq 0, \infty$.

Improving all the above results, Yi [9] proved the following theorem.

THEOREM 1.10. Let $f$ be a transcendental meromorphic function and $Q_{1}[f], Q_{2}[f]$ be two differential polynomials generated by $f$ such that $Q_{1}[f] \neq \equiv 0, Q_{2}[f] \neq \equiv 0$, and $P[f]=\sum_{j=0}^{n} a_{j} f^{j}\left(a_{n} \neq \equiv\right)$, where $a_{1}, a_{2}, \ldots, a_{n}$ are small functions of $f$. If $F=P[f]$ $Q_{1}[f]+Q_{2}[f]$, then

$$
\left(n-\gamma_{Q_{2}}\right) T(r, f) \leq \bar{N}(r, 0 ; F)+\bar{N}(r, 0 ; P[f])+\left(\Gamma_{Q_{2}}-\gamma_{Q_{2}}+1\right) \bar{N}(r, f)+S(r, f) .
$$

In Theorem 1.10 we see that the influence of $Q_{1}[f]$ on the value distribution of $F$ is ignored. In this paper, we show that Theorem 1.10 can further be improved if the influence of $Q_{1}[f]$ is taken into consideration. Throughout, we ignore zeros and poles of any small function of $f$ because the corresponding counting function is absorbed in $S(r, f)$.

2. Lemmas. In this section, we present some lemmas which will be needed in the sequel.

LEMMA 2.1 (see [4]). Let $f$ be a nonconstant meromorphic function and $Q^{*}[f], Q[f]$ denote differential polynomials generated by $f$ with arbitrary meromorphic coefficients 
$q_{1}^{*}, q_{2}^{*}, \ldots, q_{s}^{*}$ and $q_{1}, q_{2}, \ldots, q_{t}$, respectively. Further let $P[f]=\sum_{j=0}^{n} a_{j} f^{j}\left(a_{n} \neq \equiv 0\right)$ and $\gamma_{Q} \leq n$. If $P[f] Q^{*}[f]=Q[f]$, then

$$
m\left(r, Q^{*}[f]\right) \leq \sum_{j=1}^{s} m\left(r, q_{j}^{*}\right)+\sum_{j=1}^{t} m\left(r, q_{j}\right)+S(r, f) .
$$

LEMMA 2.2. Let $Q[f]=\sum_{j=1}^{l} b_{j} M_{j}[f]$ be a differential polynomial generated by $f$ of order and lower degree $k$ and $\underline{\gamma}_{Q}$, respectively. If $z_{0}$ is a zero of $f$ with multiplicity $\mu$ $(>k)$ and $z_{0}$ is not a pole of any of the coefficients $b_{j}(j=1,2, \ldots, l)$, then $z_{0}$ is a zero of $Q[f]$ with multiplicity at least $(\mu-k) \underline{\gamma}_{Q}$.

Proof. Clearly $z_{0}$ is a zero of $M_{j}[f]$ with multiplicity

$$
\begin{aligned}
\mu n_{0 j}+ & (\mu-1) n_{1 j}+\cdots+(\mu-k) n_{k j} \\
& =\mu \gamma_{M_{j}}-\left(\Gamma_{M_{j}}-\gamma_{M_{j}}\right)=(\mu-k) \gamma_{M_{j}}+(k+1) \gamma_{M_{j}}-\Gamma_{M_{j}} \\
& \geq(\mu-k) \gamma_{M_{j}} \geq(\mu-k) \underline{\gamma}_{Q} .
\end{aligned}
$$

Since $z_{0}$ is assumed not to be a pole of the coefficients $b_{j}(j=1,2, \ldots, l)$ we see that $z_{0}$ is a zero of $Q[f]$ with multiplicity at least $(\mu-k) \underline{\gamma}_{Q}$. This proves the lemma.

LEMMA 2.3 (see [1]). The following inequality holds:

$$
N(r, P[f]) \leq \gamma_{P} N(r, f)+\left(\Gamma_{P}-\gamma_{P}\right) \bar{N}(r, f)+S(r, f) .
$$

LEMmA 2.4 (see [7]). Let $P[f]=\sum_{i=0}^{n} a_{i} f^{i}$, where $a_{n}(\equiv 0), a_{n-1}, \ldots, a_{1}, a_{0}$ are small functions of $f$. Then $m(r, P[f])=n m(r, f)+S(r, f)$.

LEMMA 2.5 (see [4]). If $Q[f]$ is a differential polynomial generated by $f$ with arbitrary meromorphic coefficients $q_{j}(1 \leq j \leq n)$, then

$$
m(r, Q[f]) \leq \gamma_{Q} m(r, f)+\sum_{j=1}^{n} m\left(r, q_{j}\right)+S(r, f) .
$$

LEMMA 2.6 (see [8]). If $P[f]$ is as in Lemma 2.4, then $T(r, P[f])=n T(r, f)+S(r, f)$.

3. The main result. In this section, we present the main result of the paper.

THEOREM 3.1. Let $f$ be a transcendental meromorphic function in the open complex plane, and $Q_{1}[f]$ ( $\left.\equiv \equiv 0\right), Q_{2}[f]$ ( $\neq \equiv$ ) be two differential polynomials generated by $f$ such that $k$ and $\underline{\gamma}_{Q_{1}}$ be the order and lower degree of $Q_{1}[f]$, respectively and $P[f]=$ $\sum_{i=0}^{n} a_{i} f^{i}$, where $a_{n}(\neq \equiv), a_{n-1}, \ldots, a_{0}$ are small functions of $f$. If

$$
F=P[f] Q_{1}[f]+Q_{2}[f],
$$

then

$$
\begin{aligned}
\left(n-\gamma_{Q_{2}}\right) T(r, f) \leq & \bar{N}(r, 0 ; F)+\bar{N}(r, 0 ; P[f])+\left(\Gamma_{Q_{2}}-\gamma_{Q_{2}}+1\right) \bar{N}(r, f) \\
& -\gamma\left\{N(r, 0 ; f)-N_{k+1}(r, 0 ; f)\right\}+S(r, f),
\end{aligned}
$$

where $\gamma=\underline{\gamma}_{Q_{1}}$ if $n \geq \gamma_{Q_{2}}$ and $\gamma=0$ if $n<\gamma_{Q_{2}}$. 
Proof. If $n<\gamma_{Q_{2}}$, the theorem is obvious. So we suppose that $n \geq \gamma_{Q_{2}}$. Differentiating (3.1) we get

$$
F^{\prime}=P^{\prime}[f] Q_{1}[f]+P[f] Q_{1}^{\prime}[f]+Q_{2}^{\prime}[f]
$$

where $P^{\prime}[f]=(d / d z) P[f]$ and $Q_{i}^{\prime}[f]=(d / d z) Q_{i}[f]$ for $i=1,2$.

Multiplying (3.1) by $\left(F^{\prime} / F\right)$, and substituting in (3.3) we get

$$
P[f] Q^{*}[f]=Q[f],
$$

where

$$
\begin{aligned}
Q^{*}[f] & =\left(\frac{F^{\prime}}{F}-\frac{P^{\prime}[f]}{P[f]}\right) Q_{1}[f]-Q_{1}^{\prime}[f], \\
Q[f] & =Q_{2}^{\prime}[f]-\left(\frac{F^{\prime}}{F}\right) Q_{2}[f] .
\end{aligned}
$$

First we suppose that $Q^{*}[f] \not \equiv 0$. By Lemma 2.1, it follows from (3.4) that $m(r$, $\left.Q^{*}[f]\right)=S(r, f)$ because $\gamma_{Q}=\gamma_{Q_{2}} \leq n$.

Since $P[f]=Q[f] / Q^{*}[f]$, we get by Lemma 2.5 and the first fundamental theorem

$$
\begin{aligned}
m(r, P[f]) & \leq m(r, Q[f])+m\left(r, 0 ; Q^{*}[f]\right) \\
& \leq \gamma_{Q_{2}} m(r, f)+m\left(r, Q^{*}[f]\right)+N\left(r, Q^{*}[f]\right)-N\left(r, 0 ; Q^{*}[f]\right)+S(r, f) \\
& =\gamma_{Q_{2}} m(r, f)+N\left(r, Q^{*}[f]\right)-N\left(r, 0 ; Q^{*}[f]\right)+S(r, f) .
\end{aligned}
$$

So by Lemma 2.4

$$
\left(n-\gamma_{Q_{2}}\right) m(r, f) \leq N\left(r, Q^{*}[f]\right)-N\left(r, 0 ; Q^{*}[f]\right)+S(r, f) .
$$

From (3.5) we see that possible poles of $Q^{*}[f]$ occur at the poles of $f$ and zeros of $F$ and $P[f]$. Also we note that the zeros of $F$ and $P[f]$ are at most simple poles of $Q^{*}[f]$. Let $z_{0}$ be a pole of $f$ with multiplicity $\mu$. Then $z_{0}$ is a pole of $Q[f]$ with multiplicity not exceeding $(\mu-1) \gamma_{Q_{2}}+\Gamma_{Q_{2}}+1=\mu \gamma_{Q_{2}}+\Gamma_{Q_{2}}-\gamma_{Q_{2}}+1$ and $z_{0}$ is a pole of $P[f]$ with multiplicity $n \mu$. Hence, from (3.4) it follows that $z_{0}$ is a pole of $Q^{*}[f]$ with multiplicity not exceeding $\mu \gamma_{Q_{2}}+\Gamma_{Q_{2}}-\gamma_{Q_{2}}+1-n \mu=\Gamma_{Q_{2}}-\gamma_{Q_{2}}+1-\left(n-\gamma_{Q_{2}}\right) \mu$. Therefore

$$
\begin{aligned}
N\left(r, Q^{*}[f]\right) \leq & \bar{N}(r, 0 ; F)+\bar{N}(r, 0 ; P[f])+\left(\Gamma_{Q_{2}}-\gamma_{Q_{2}}+1\right) \bar{N}(r, f) \\
& -\left(n-\gamma_{Q_{2}}\right) N(r, f)+S(r, f) .
\end{aligned}
$$

Now we note that the order of the differential polynomial $Q_{1}^{\prime}[f]$ is $k+1$. Let $z_{0}$ be a zero of $f$ with multiplicity $\mu>k+1$. Let $\underline{\gamma}_{Q_{1}} \geq 1$. Then by Lemma 2.2, we see that $z_{0}$ is a zero of $Q_{1}[f]$ with multiplicity at least $(\mu-1) \underline{\gamma}_{Q_{1}}$. Also $z_{0}$ may be a pole of $\left(F^{\prime} / F\right)-P^{\prime}[f] / P[f]$ with multiplicity not exceeding 1 . So $z_{0}$ is a zero of $\left(\left(F^{\prime} / F\right)-\right.$ $\left.P^{\prime}[f] / P[f]\right) Q_{1}[f]$ with multiplicity at least $(\mu-k) \underline{\gamma}_{Q_{1}}-1$. 
Since the lower degree of $Q_{1}^{\prime}[f]$ is $\underline{\gamma}_{Q_{1}}$, it follows from Lemma 2.2 that $z_{0}$ is a zero of $Q_{1}^{\prime}[f]$ with multiplicity at least $(\mu-k-1) \underline{\gamma}_{Q_{1}}$.

Therefore $z_{0}$ is a zero of $Q^{*}[f]$ with multiplicity at least $(\mu-k-1) \underline{\gamma}_{Q_{1}}$. Hence

$$
\begin{aligned}
N\left(r, 0 ; Q^{*}[f]\right) \\
\quad \geq N\left(r, 0 ; Q^{*}[f] \mid f=0,>k+1\right) \\
\quad \geq \underline{\gamma}_{Q_{1}} N(r, 0 ; f \mid>k+1)-\underline{\gamma}_{Q_{1}}(k+1) \bar{N}(r, 0 ; f>k+1)+S(r, f) \\
\quad=\underline{\gamma}_{Q_{1}} N(r, 0 ; f)-\underline{\gamma}_{Q_{1}}\{N(r, 0 ; f \mid \leq k+1)+(k+1) \bar{N}(r, 0 ; f \mid>k+1)\}+S(r, f) .
\end{aligned}
$$

So

$$
N\left(r, 0 ; Q^{*}[f]\right) \geq \underline{\gamma}_{Q_{1}}\left\{N(r, 0 ; f)-N_{k+1}(r, 0 ; f)\right\}+S(r, f) .
$$

If $\underline{\gamma}_{Q_{1}}=0$, inequality (3.11) obviously holds. Now from (3.8), (3.9), and (3.11) we get

$$
\begin{aligned}
\left(n-\gamma_{Q_{2}}\right) T(r, f) \leq & \bar{N}(r, 0 ; F)+\bar{N}(r, 0 ; P[f])+\left(\Gamma_{Q_{2}}-\gamma_{Q_{2}}+1\right) \bar{N}(r, f) \\
& -\underline{\gamma}_{Q_{1}}\left\{N(r, 0 ; f)-N_{k+1}(r, 0 ; f)\right\}+S(r, f) .
\end{aligned}
$$

Next we suppose that $Q^{*}[f] \equiv 0$. Then from (3.4) it follows that $Q[f] \equiv 0$, and so using (3.1) we get $P[f] Q_{1}[f]=c Q_{2}[f]$, where $c$ is a nonzero constant. Then in a similar line of calculation for inequalities (3.8), (3.9), and (3.11) we get

$$
\begin{aligned}
\left(n-\gamma_{Q_{2}}\right) m(r, f) & \leq N\left(r, Q_{1}[f]\right)-N\left(r, 0 ; Q_{1}[f]\right)+S(r, f), \\
N\left(r, Q_{1}[f]\right) & \leq\left(\Gamma_{Q_{2}}-\gamma_{Q_{2}}+1\right) \bar{N}(r, f)-\left(n-\gamma_{Q_{2}}\right) N(r, f)+S(r, f), \\
N\left(r, 0 ; Q_{1}[f]\right) & \geq \underline{\gamma}_{Q_{1}}\left\{N(r, 0 ; f)-N_{k+1}(r, 0 ; f)\right\}+S(r, f) .
\end{aligned}
$$

Now from (3.13) we get

$$
\begin{aligned}
\left(n-\gamma_{Q_{2}}\right) T(r, f) \leq & \bar{N}(r, 0 ; F)+\bar{N}(r, 0 ; P[f])+\left(\Gamma_{Q_{2}}-\gamma_{Q_{2}}+1\right) \bar{N}(r, f) \\
& -\underline{\gamma}_{Q_{1}}\left\{N(r, 0 ; f)-N_{k+1}(r, 0 ; f)\right\}+S(r, f) .
\end{aligned}
$$

This proves the theorem.

REMARK 3.2. The following example shows that Theorem 3.1 is sharp.

EXAMPLE 3.3. Let $f=e^{z}-2, P[f]=f+2, Q_{1}[f]=f$, and $Q_{2}[f]=1$. Then $F=P[f] Q_{1}[f]+Q_{2}[f]=\left(e^{z}-1\right)^{2}$ and $k=0, \underline{\gamma}_{Q_{1}}=1, \gamma_{Q_{2}}=0, n=1$. Also we see that

$$
\begin{aligned}
\left(n-\gamma_{Q_{2}}\right) T(r, f)= & \bar{N}(r, 0 ; F)+\bar{N}(r, 0 ; P[f])+\left(\Gamma_{Q_{2}}-\gamma_{Q_{2}}+1\right) \bar{N}(r, f) \\
& -\underline{\gamma}_{Q_{1}}\left\{N(r, 0 ; f)-N_{k+1}(r, 0 ; f)\right\}+S(r, f) .
\end{aligned}
$$

4. Applications. As applications of Theorem 1.10, Yi [9] proved the following theorems which improve Theorems 1.8 and 1.9. 
THEOREM 4.1. Let $f$ be a transcendental meromorphic function and $Q_{1}[f](\not \equiv 0)$, $Q_{2}[f](\equiv 0)$ be two differential polynomials generated by $f$. Let $F=f^{n} Q_{1}[f]+Q_{2}[f]$ and

$$
\limsup _{r \rightarrow \infty} \frac{\bar{N}(r, 0 ; f)+\left(\Gamma_{Q_{2}}-\gamma_{Q_{2}}+1\right) \bar{N}(r, f)}{T(r, f)}<n-\gamma_{Q_{2}}
$$

Then $\Theta(a ; F)<1$ for any small function $a\left(\not \equiv \infty, Q_{2}[f]\right)$ of $f$.

THEOREM 4.2. Let $F=f^{n} Q[f]$, where $Q[f]$ is a differential polynomial generated by $f$ and $Q[f] \not \equiv$. If

$$
\limsup _{r \rightarrow \infty} \frac{\bar{N}(r, 0 ; f)+\bar{N}(r, f)}{T(r, f)}<n,
$$

then $\Theta(a ; F)<1$, where $a(\not \equiv 0, \infty)$ is a small function of $f$.

Considering the following examples, Yi [9] claimed that Theorems 4.1 and 4.2 are sharp.

EXAMPLE 4.3. Let $f=\left(e^{4 z}+1\right) /\left(e^{4 z}-1\right), Q_{1}[f]=1, Q_{2}[f]=f^{\prime}-1$, and $F=$ $f^{4} Q_{1}[f]+Q_{2}[f]$. Then $n=4, \gamma_{Q_{2}}=1, \Gamma_{Q_{2}}=2$, and

$$
\limsup _{r \rightarrow \infty} \frac{\bar{N}(r, 0 ; f)+\left(\Gamma_{Q_{2}}-\gamma_{Q_{2}}+1\right) \bar{N}(r, f)}{T(r, f)}=n-\gamma_{Q_{2}} .
$$

Also we see that $\Theta(0 ; F)=1$.

EXAMPLE 4.4. Let $f=\left(e^{z}-1\right) /\left(e^{z}+1\right), Q_{1}[f]=1, F=f^{n} Q_{1}[f]$, where $n=2$. It is easy to verify that

$$
\limsup _{r \rightarrow \infty} \frac{\bar{N}(r, 0 ; f)+\bar{N}(r, f)}{T(r, f)}=n
$$

and $\Theta(1 ; F)=1$.

The following examples suggest that some improvements of Theorems 4.1 and 4.2 are possible.

EXAMPLE 4.5. Let $f=\left(\left(e^{z}-1\right) /\left(e^{z}+1\right)\right)^{2}, Q_{1}[f]=f, Q_{2}[f]=1$, and $F=f Q_{1}[f]+$ $Q_{2}[f]$. Then $n=1, \underline{\gamma}_{Q_{1}}=1, \gamma_{Q_{2}}=0, \Gamma_{Q_{2}}=0$, and the order of the differential polynomial $Q_{1}[f]$ is zero. Clearly

$$
\limsup _{r \rightarrow \infty} \frac{\bar{N}(r, 0 ; f)+\left(\Gamma_{Q_{2}}-\gamma_{Q_{2}}+1\right) \bar{N}(r, f)}{T(r, f)}=n-\gamma_{Q_{2}} .
$$

Also we see that $\Theta(1 ; F)=\Theta(\infty ; F)=3 / 4, \Theta(2 ; F)=1 / 2$ and so, by Nevanlinna's three small functions theorem (cf. [6, page 47]), $\Theta(a ; F) \leq 2-3 / 4-1 / 2=3 / 4$ for any small function $a(\equiv 1,2, \infty)$. However, we note that

$$
\limsup _{r \rightarrow \infty} \frac{\bar{N}(r, 0 ; f \mid \leq 1)+\left(\Gamma_{Q_{2}}-\gamma_{Q_{2}}+1\right) \bar{N}(r, f)}{T(r, f)}=\frac{1}{2}<n-\gamma_{Q_{2}} .
$$


EXAMPLE 4.6. Let $f=\left(\left(e^{z}-1\right) /\left(e^{z}+1\right)\right)^{2}, Q[f]=f$, and $F=f Q[f]$. Then $n=1$, $\underline{\gamma}_{Q}=1$, and the order of the differential polynomial $Q[f]$ is zero. Clearly

$$
\limsup _{r \rightarrow \infty} \frac{\bar{N}(r, 0 ; f)+\bar{N}(r, f)}{T(r, f)}=n
$$

and $\Theta(a ; F)<1$ for any small function $a$ of $f$. We note that

$$
\limsup _{r \rightarrow \infty} \frac{\bar{N}(r, 0 ; f \mid \leq 1)+\bar{N}(r, f)}{T(r, f)}=\frac{1}{2}<n .
$$

The following two theorems improve Theorems 4.1 and 4.2.

THEOREM 4.7. Let $f$ be a transcendental meromorphic function and $Q_{1}[f], Q_{2}[f]$ be two differential polynomials generated by $f$ which are not identically zero. Let $F=$ $f^{n} Q_{1}[f]+Q_{2}[f]$. If

$$
\limsup _{r \rightarrow \infty} \frac{\bar{N}\left(r, 0 ; f \mid \leq \chi_{Q_{1}}\right)+\left(\Gamma_{Q_{2}}-\gamma_{Q_{2}}+1\right) \bar{N}(r, f)}{T(r, f)}<n-\gamma_{Q_{2}},
$$

then $\Theta(a ; F)<1$ for any small function $a\left(\equiv \infty, Q_{2}[f]\right)$ of $f$, where

$$
\chi_{Q_{1}}= \begin{cases}1+k & \text { if } \underline{\gamma}_{Q_{1}} \geq 1, \\ \infty & \text { if } \underline{\underline{\gamma}}_{Q_{1}}=0,\end{cases}
$$

and $k$ is the order of the differential polynomial $Q_{1}[f]$.

THEOREM 4.8. Let $f$ be a transcendental meromorphic function and $Q[f](\not \equiv 0)$ be a differential polynomial generated by $f$. If $F=f^{n} Q[f]$ and

$$
\limsup _{r \rightarrow \infty} \frac{\bar{N}\left(r, 0 ; f \mid \leq \chi_{Q}\right)+\bar{N}(r, f)}{T(r, f)}<n,
$$

then $\Theta(a ; F)<1$ for every small function $a(\not \equiv 0, \infty)$ of $f$, where

$$
\chi_{Q}= \begin{cases}1+k & \text { if } \underline{\gamma}_{Q} \geq 1, \\ \infty & \text { if } \underline{\gamma}_{Q}=0,\end{cases}
$$

and $k$ is the order of the differential polynomial $Q[f]$.

REMARK 4.9. Theorem 4.7 improves Theorems 1.8 and 4.1, and Theorem 4.8 improves Theorems 1.9 and 4.2 .

REMARK 4.10. The following examples show that Theorems 4.7 and 4.8 are sharp.

EXAMPLE 4.11. Let $f=e^{z}-1, Q_{1}[f]=f^{\prime}-f, Q_{2}[f]=2 f^{\prime}$, and $F=f^{2} Q_{1}[f]+$ $Q_{2}[f]$. Then $n=2, k=1, \Gamma_{Q_{2}}=2, \gamma_{Q_{2}}=1$, and

$$
\limsup _{r \rightarrow \infty} \frac{\bar{N}(r, 0 ; f \mid \leq 2)+\left(\Gamma_{Q_{2}}-\gamma_{Q_{2}}+1\right) \bar{N}(r, f)}{T(r, f)}=n-\gamma_{Q_{2}} .
$$

Also we see that $\Theta(1 ; F)=1$. 
EXAMPLE 4.12. Let $f=e^{z}+1, Q[f]=f-f^{\prime}$, and $F=f Q[f]$. Then $\underline{\gamma}_{Q}=1, k=1$, $n=1$, and

$$
\limsup _{r \rightarrow \infty} \frac{\bar{N}(r, 0 ; f \mid \leq 2)+\bar{N}(r, f)}{T(r, f)}=n
$$

Also we see that $\Theta(1 ; F)=1$.

As other applications of Theorem 3.1, we obtain the following results which improve Theorems 1.6 and 1.7.

THEOREM 4.13. Let $f$ be a transcendental meromorphic function, and $F=f^{\prime}-a f^{n}$, where $a(\equiv 0)$ is a small function of $f$. If $n(\geq 5)$ is an integer, then $\Theta(b ; F) \leq 4 / n$ for any small function $b(\equiv \equiv)$ of $f$.

THEOREM 4.14. Let $f$ be a transcendental meromorphic function. If $F=f^{n} f^{\prime}$ and $n(\geq 3)$ is an integer, then $\Theta(a ; F) \leq 4 /(n+2)$ for any small function $a(\equiv \equiv, \infty)$ of $f$.

We prove Theorems 4.8 and 4.14 only.

Proof OF TheOrem 4.8. First we treat the case $\underline{\gamma}_{Q} \geq 1$. Then by Theorem 3.1 we get

$$
\begin{aligned}
n T(r, f) \leq & \bar{N}(r, a ; F)+\bar{N}(r, 0 ; P[f])+\bar{N}(r, f) \\
& -\underline{\gamma}_{Q}\left\{N(r, 0 ; f)-N_{k+1}(r, 0 ; f)\right\}+S(r, f) \\
\leq & \bar{N}(r, a ; F)+\bar{N}(r, 0 ; f)-N(r, 0 ; f) \\
& +N_{k+1}(r, 0 ; f)+\bar{N}(r, f)+S(r, f),
\end{aligned}
$$

that is,

$$
n T(r, f) \leq \bar{N}(r, a ; F)+\bar{N}(r, 0 ; f \mid \leq k+1)+\bar{N}(r, f)+S(r, f) .
$$

Now we treat the case $\underline{\gamma}_{Q}=0$. Then from Theorem 3.1 we get

$$
n T(r, f) \leq \bar{N}(r, a ; F)+\bar{N}(r, 0 ; f)+\bar{N}(r, f)+S(r, f) .
$$

Combining (4.16) and (4.17), we obtain

$$
n T(r, f) \leq \bar{N}(r, a ; F)+\bar{N}\left(r, 0 ; f \mid \leq \chi_{Q}\right)+\bar{N}(r, f)+S(r, f)
$$

from which the theorem follows.

Proof OF TheOrem 4.14. Proceeding in the line of the proof of Theorem 4.8 we get

$$
n T(r, f) \leq \bar{N}(r, a ; f)+\bar{N}(r, 0 ; f \mid \leq k+1)+\bar{N}(r, f)+S(r, f),
$$

that is,

$$
(n-2) T(r, f) \leq \bar{N}(r, a ; F)+S(r, f) .
$$


Now by Lemmas 2.3 and 2.5 we see that

$$
T(r, F) \leq(n+2) T(r, f)+S(r, f) .
$$

If possible let $\Theta(a ; F)>4 /(n+2)$. Then there exits an $\varepsilon(>0)$ such that for all large values of $r$

$$
\bar{N}(r, a ; F)<\left(\frac{n-2}{n+2}-\varepsilon\right) T(r, F) .
$$

From (4.20), (4.21), and (4.22) we get

$$
\varepsilon(n+2) T(r, f) \leq S(r, f),
$$

which is a contradiction. This proves the theorem.

\section{REFERENCES}

[1] N. R. Bhattacharjee and I. Lahiri, Growth and value distribution of differential polynomials, Bull. Math. Soc. Sci. Math. Roumanie (N.S.) 39 (1996), no. 1-4, 85-104. Zbl 892.30029.

[2] C. Chuang, Une généralisation d'une inégalité de Nevanlinna, Sci. Sinica 13 (1964), 887895 (French). MR 30\#2148. Zbl 146.10202.

[3] J. Clunie, On a result of Hayman, J. London Math. Soc. 42 (1967), 389-392. MR 35\#5618. Zbl 169.40801.

[4] W. Doeringer, Exceptional values of differential polynomials, Pacific J. Math. 98 (1982), no. 1, 55-62. MR 83a:30032. Zbl 469.30023.

[5] W. K. Hayman, Picard values of meromorphic functions and their derivatives, Ann. of Math. (2) 70 (1959), 9-42. MR 22\#1675. Zbl 088.28505.

[6] Meromorphic Functions, Oxford Mathematical Monographs, Clarendon Press, Oxford, 1964. MR 29\#1337. Zbl 115.06203.

[7] C. C. Yang, On deficiencies of differential polynomials, Math. Z. 116 (1970), 197-204. MR 42\#3279. Zbl 194.38101.

[8] _ On deficiencies of differential polynomials. II, Math. Z. 125 (1972), 107-112. MR 45\#3710. Zbl 217.38402.

[9] H. X. Yi, On the value distribution of differential polynomials, J. Math. Anal. Appl. 154 (1991), no. 2, 318-328. MR 92f:30045. Zbl 725.30022.

[10] Z. Zhang and W. Li, Tumura-Clunie's theorem for meromorphic functions, Southeast Asian Bull. Math. 20 (1996), no. 4, 9-14. MR 97m:30044. Zbl 903.30022.

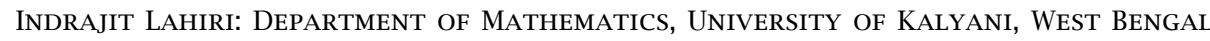
741235, INDIA

E-mail address: indrajit@ca12.vsn1.net. in 


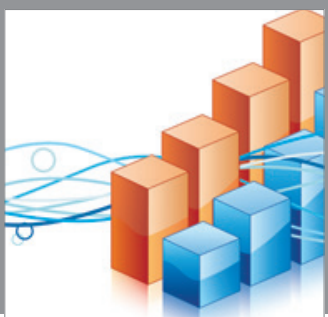

Advances in

Operations Research

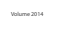

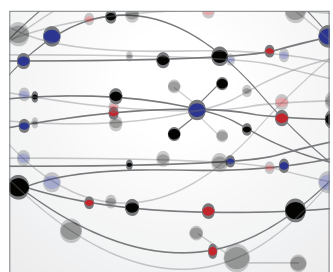

\section{The Scientific} World Journal
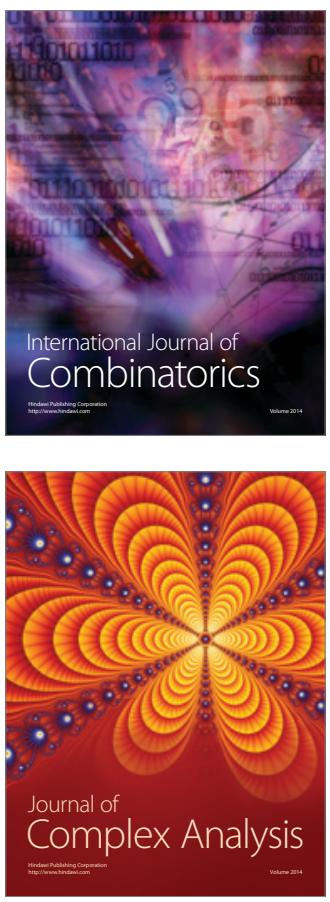

International Journal of

Mathematics and

Mathematical

Sciences
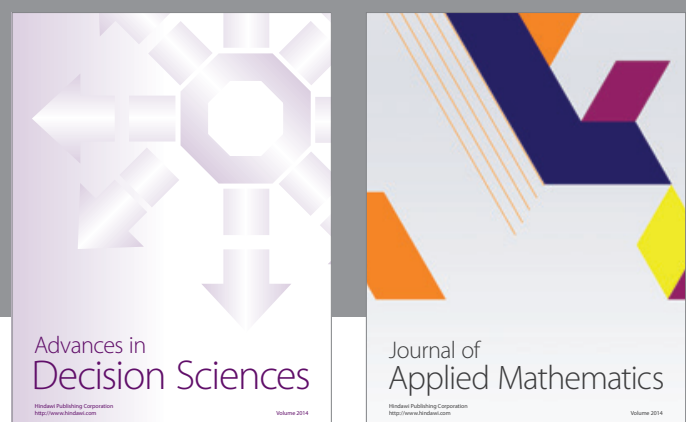

Journal of

Applied Mathematics
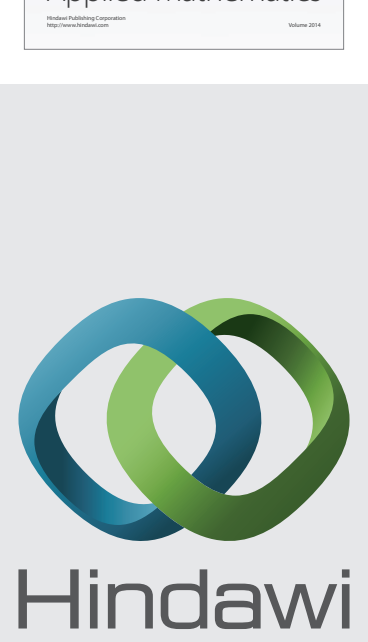

Submit your manuscripts at http://www.hindawi.com
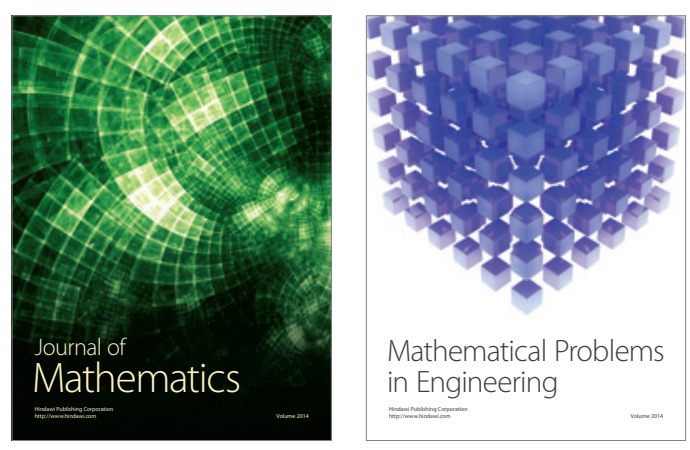

Mathematical Problems in Engineering
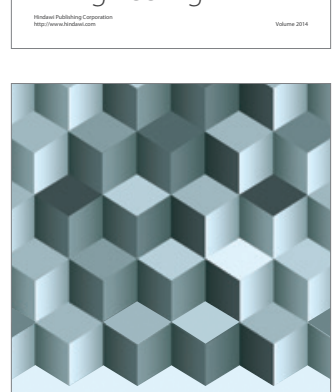

Journal of

Function Spaces
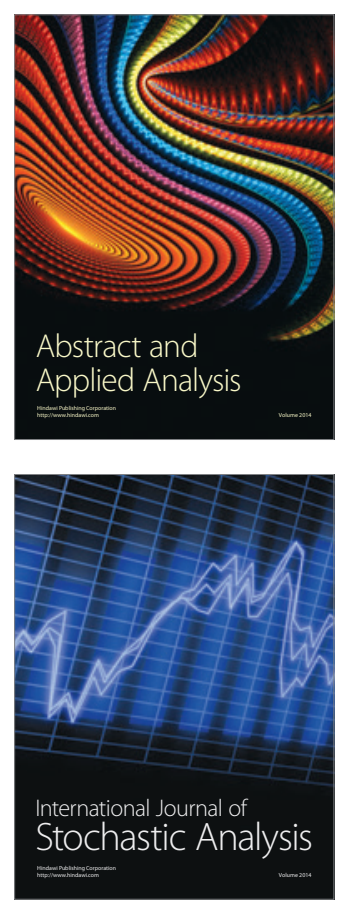

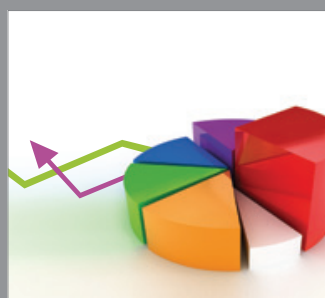

ournal of

Probability and Statistics

Promensencen
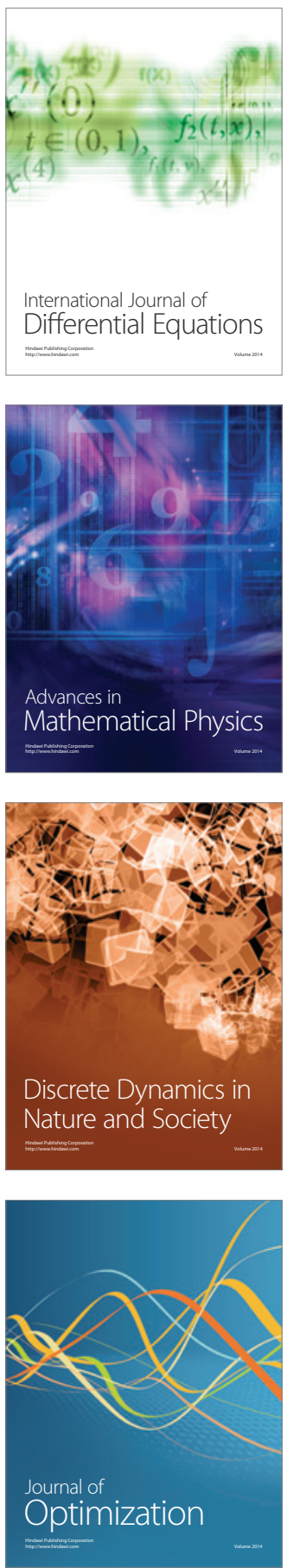